\title{
Análisis multivariado de indicadores socioeconómico-productivos del ganado caprino-ovino en el departamento de La Guajira, Colombia
}

Recibido:

15 de marzo de 2008

Aceptado:

23 de octubre de 2008

\section{Resumen}

Los modelos y métodos estadísticos multivariados se convierten en una herramienta fundamental, para el análisis y evaluación del sistema de produccióncomercialización del ganado caprino-ovino en el departamento de La Guajira, siendo su objetivo caracterizar, desde el punto de vista socio-económico, el desarrollo de la producción e identificar los factores que han incidido en el débil desarrollo de esta especie. En el departamento de la Guajira la especie caprinaovina representa el 60\% de la dieta cárnica de sus habitantes, constituyendo una importante fuente de ingresos económicos para la etnia wayuú, productores en potencia del ganado menor. Se realizó una investigación de tipo exploratoriodescriptivo, mediante el uso del diseño no experimental de corte transversal, a través de los software Statgraphics Plus versión 5.1 (2001) y SPSS versión 13.0 (2004). La información se recogió mediante la aplicación de 334, durante el periodo de julio 2005 a julio de 2006. Los resultados del análisis multivariado muestran que es bajo, el número de animales con problemas genéticos y de lactancia. La producción de leche por día animal es de 0.61 litros, produciendo el 75\% de ellos 1 litro/día/animal. La incidencia de enfermedades del ganado es en épocas de invierno y verano, predominando la época de invierno. Además de los indicadores económicos se seleccionaron 6 componentes principales que representan el $60.146 \%$ y de las 9 variables productivas se seleccionaron 4 componentes principales que representan el $61.821 \%$ de la variación total ya que tienen un auto valor superior a 1.0.

Palabras clave: Análisis multivariado, caprino-ovino, La Guajira.

\section{Abstract}

Multivariety of models and statistics methods have become a key tool for testing and analyzing the state production and marketing system of ovine-caprine in the of La Guajira (Colombia). One of the goals is to characterize. From the social economic view, the development of the production and identification of factors that have affected in this cattle's weak development. The specie ovinecaprine represents a $60 \%$ of the meat diet in the inhabitants from the state of LA GUAJIRA, it represents an important economic source of income to the wayuu ethnic, which are the fewer producers of cattle. The research is projected as an
Especialita en de la Guajira-Colombia. atenaida03@yahoo.es

2Licenciada en matemática.

Especialista en estadística matemática. Doctora en ciencias.

Universidad Agraria de la Habana, Cuba

caridadwalkiria@yahoo.es

${ }^{3}$ Licenciada en matemática.

Master en ciencias en la

especialidad de matemática

aplicada. Doctora en ciencias veterinarias. Universidad Agraria de La Habana, Cuba. lucia@isch.edu.cu 
alternative to improve the demand and offer of the production in the region. It has been done a research from the Descriptive-explanatory type through the use of The Design Unexperimental of Transversal Cut using the software Statgraphics Plus version 5.1 (2001) and SPSS 13.0(2004), with the purpose to describe variables and analyze its affection and interrelation in a given time. The information has been gathered through the appliance of 334 polls distributed in four sceneries: producer from the wayuú ethnic, transporters, intermediaries and consumers of the ovine-caprine cattle, in the municipality of Riohacha, during the period of time of July 2005 to July 2006. The result of the multi-varied analysis shows the low number, of genetic problems and lacting in animals. The production of milk per animal is 0,61 litre. The impact of cattle illness shows in summer and winter season, hisher in winter time. It has been formulated generals, productive and economics indicators. It has been highlighted in the multi-varied analysis the sales and purchase indicators, those from the genetic type and those from the animals health.

Keywords: Multivaried Analysis, Caprine-Ovino, La Guajira.

\section{Introducción}

$\mathrm{L}$ a producción caprina-ovina ha progresado rápidamente en los últimos diez años en el departamento de La Guajira, y en particular en el municipio de Riohacha área en estudio.

La etnia Wayuú tradicionalmente se ha dedicado a la actividad del pastoreo, cría y sacrificio del ganado caprino-ovino, sin aplicación de nuevas tecnologías, mediante el uso de técnicas primitivas que se encargan de satisfacer las necesidades básicas de las familias de esta región. La especie caprina-ovina en el departamento de La Guajira, representa el $60 \%$ de la dieta cárnica de sus habitantes, constituyendo una importante fuente de ingresos económicos (Márquez, 2003-2006).

La técnica de encuestas, es una herramienta muy importante en la recolección de información. (García, 2005), plantea que en los últimos años las encuestas sobre los temas más diversos, se encuentran continuamente en los medios de comunicaciones audiovisuales y escritos.

El objetivo de este trabajo, es diagnosticar y evaluar el desarrollo socio-económico- productivo del ganado caprino-ovino y el impacto que genera en el departamento de La Guajira, Colombia.

\section{Materiales y métodos}

Según Berenson, (1996) y Lind et al. (2001), la población está formada por cuatro escenarios así: la primera población corresponde a $\mathrm{N} 1=$ 216 rancherías ó viviendas de indígenas Wayuú con asiento en el municipio de Riohacha; N2= 120 transportadores; N3= 150 intermediarios (compradores mayoristas y minoristas). La información de las tres poblaciones anteriores fue suministrada por la Unidad de Asuntos Indígenas (2006), N4 = 117.527 habitantes del municipio de RiohachaColombia, relaciona los consumidores de la carne caprina-ovina, de esta localidad, (Departamento Administrativo Nacional de Estadística en Colombia DANE, 2004).

Según Martínez (2003); Fernández y Ruiz (2006), el diseño de cada muestra para las salidas de campo quedó establecida así: $\mathrm{n}_{1}=$ 109 familias de indígenas Wayuú; $\mathrm{n}_{2}=70$ transportadores, $\mathrm{n}_{3}=55$ intermediarios, $\mathrm{y}$ 
Análisis multivariado de indicadores socioeconómico-productivos del ganado caprino-ovino en el departamento de La Guajira, Colombia
Respuestas

Año 13

No. 2

Diciembre 2008 ISSN 0122-820X $\mathrm{n}_{4}=100$ consumidores del ganado caprinoovino.

La información se recogió mediante la realización de 334 encuestas en cuatro escenarios. Para el diseño de cada muestra, se utilizó la técnica del muestreo aleatorio simple para estimar las proporciones de éxitos y fracasos (pyq), que en este caso no se conocen, sin embargo se puede considerar $(\mathrm{p}=\mathrm{q}=$ $50 \%$ ); además $\mathrm{P}=95 \%$; es la probabilidad de confianza; $\alpha=5 \%$ nivel de significación; para un área bajo la curva normal de: $\mathrm{A}=0,4750 \rightarrow$ $\mathrm{Z}=1,96$ que es la desviación estándar que se toma con respecto al nivel de confianza en la tabla de la distribución normal. De igual forma, se tomaron márgenes de errores establecidos para cada diseño de muestras a partir de un promedio de estimativo: no.

De las 334 encuestas realizadas en el trabajo de campo, se aplicaron 109 encuestas dirigidas a familias de indígenas Wayuú, de las cuales se obtuvieron 14 variables generales-económicas y 9 variables productivas, registradas en las tablas: (5),(6) y (9). La investigación realizada es de tipo exploratorio-descriptiva ya que se aborda por primera vez, buscando especificar las propiedades más importantes de esta especie en lo socio-económico y productivo. Como método de investigación, se utilizó el diseño no experimental de corte transversal, puesto que el objetivo de la investigación fué captar información en un tiempo único, con el propósito de describir variables y analizar su incidencia e interrelación en un momento dado. (Hernández-Sampieri et al, 2003 y Tamayo, 2003). Los resultados de las encuestas se procesaron mediante tablas de frecuencia univariadas y bivariadas (tablas de contingencia) y gráficos de barras. Se determinaron estadígrafos de posición y variación. Se aplicó la prueba Chi-cuadrado de independencia para tablas de contingencia y la técnica estadística multivariada de análisis de componentes principales (Bouza y Sistachs, 2002; Härdle y Simar, 2003).

La conveniencia del uso del análisis de componentes principales, se analizó mediante el contraste de esfericidad de Bartlett, que proporciona la probabilidad estadística de que la matriz de correlación de las variables, sea una matriz identidad (Hair et al., 1999; SPSS versión 13.0, 2004). La información fué procesada utilizando el tabulador electrónico Excel y los software sobre Windows: Statgraphics Plus versión 5.1 (2001) y SPSS versión 13.0 (2004).

\section{Resultados y discusión}

Las técnicas y procedimientos de recolección de la información, originaron la aplicación de 334 encuestas para el trabajo de campo. Sin embargo para el análisis de los indicadores productivos, se diseño una muestra aleatoria simple de 109 encuestas dirigidas a productores indígenas Wayuú del caprino-ovino. Después de codificadas estas encuestas se tabularon y se confeccionaron matrices de datos entre otros, localizando, 12 tipos de familias o castas con asiento en el municipio de Riohacha, productoras del ganado caprino-ovino así: 1. Epinayú; 2. Epiayú; 3. Ipuana; 4. Uriana; 5. Apshana; 6. Jarariyú; 7. Arphusana; 8. Pushaina; 9. Jusayú; 10. Wouriyú; 11.Sijona; 12. Sapuana.

En la tabla 1, se puede observar que el promedio de hijos por familias o castas en cada una de las rancherías de indígenas Wayuú, es 7 y el valor más frecuente es 8 hijos. Sin embargo el $25 \%$ de las familias o castas tiene hasta 4 hijos, el $50 \%$ hasta 7 hijos y el 75\% hasta 9 hijos. Es apreciable la dispersión que se presenta en este indicador, que concuerda con el rango de variación del mismo.
Tabla 1. Estadígrafo de posición y dispersión del número de hijos por familia o casta

\begin{tabular}{|l|c|}
\hline \multicolumn{1}{|c|}{ Resumen estadístico } & Número de hijos por familia \\
\hline Media aritmética & 6,54 \\
\hline Mediana(Segundo cuartil) & 7,00 \\
\hline Moda & 8,00 \\
\hline Varianza & 13,62 \\
\hline Desviación típica & 3,69 \\
\hline Mínimo & 0,00 \\
\hline Máximo & 17,00 \\
\hline Rango & 17,00 \\
\hline Primer cuartil & 4,00 \\
\hline Tercer cuartil & 9,00 \\
\hline Coeficiente de variación & $56,45 \%$ \\
\hline
\end{tabular}


Análisis multivariado de indicadores socioeconómico-productivos del ganado caprino-ovino en el departamento de La Guajira, Colombia
De los resultados de la tabla 2 , que recoge la aplicación de la prueba Chi-cuadrado $(\chi)^{2}$ de independencia, se puede inferir que no existe dependencia (con $p=0,3698$ ), entre la familia o casta y el número de animales que producen para el consumo humano, aunque la figura 1, muestra que de las doce familias o castas analizadas, la 1; 3; 4 y 7 (correspondientes a las familias o castas: Epinayú, Ipuana, Uriana y Pushaina), son las mayores productoras del caprino-ovino en el municipio de Riohacha, departamento de La Guajira.

Tabla 2. Análisis de la relación entre la familia o casta y el número de animales que producen para el consumo humano.

\begin{tabular}{|c|c|c|}
\hline Estadístico $(\chi)^{2}$ & Grados de libertad & p valor \\
\hline 23,58 & 22 & 0,3698 \\
\hline
\end{tabular}

Figura 1. Familias o castas y animales que producen.

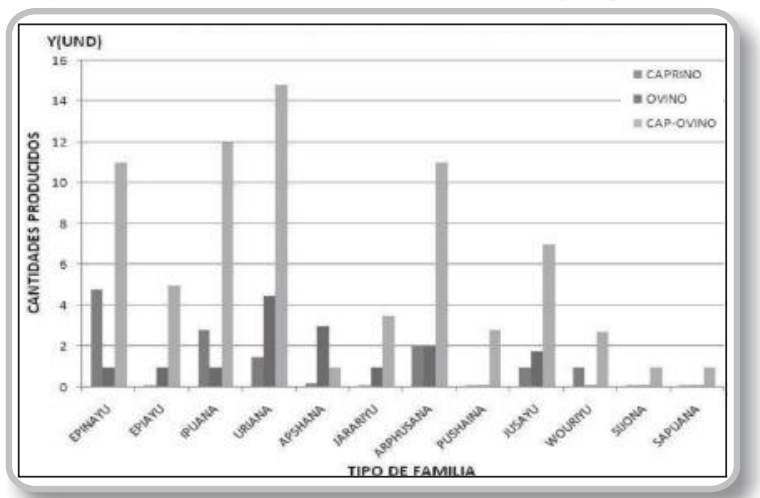

En la tabla 3, se puede apreciar que el contraste de Bartlett, indica que es muy improbable $(p=0,004)$ que la matriz de correlación de las variables, sea una matriz identidad. Teniendo en cuenta este resultado, se considera que resulta adecuado plantear el uso de la técnica estadística multivariada, del análisis de componentes principales, bajo las condiciones naturales de producción (rancherías), en que se desarrolló este estudio.

Tabla 3. Resultados del contraste de esfericidad de Bartlett.

\begin{tabular}{|c|c|c|}
\hline $\begin{array}{c}\text { Prueba de } \\
\text { esfericidad de } \\
\text { Bartlett }\end{array}$ & $\begin{array}{c}\text { Chi-cuadrado } \\
\text { aproximado }\end{array}$ & 131,513 \\
\cline { 2 - 3 } & Grados de libertad & 91 \\
\cline { 2 - 3 } & Significación. & 0,004 \\
\hline
\end{tabular}

De las 14 variables generales y económicas se seleccionaron 6 componentes principales, que representan el 60,146\% de la variación total, ya que tienen un auto valor superior a 1.0, lo que resulta aceptable bajo las condiciones estudiadas (tabla 4).

Tabla 4. Resultados de la aplicación del análisis de componentes principales.

\begin{tabular}{|c|c|c|c|}
\hline \multirow[t]{2}{*}{ Componente } & \multicolumn{3}{|c|}{ Autovalores iniciales } \\
\hline & Total & $\begin{array}{l}\% \text { de la } \\
\text { varianza }\end{array}$ & $\begin{array}{c}\% \\
\text { acumulado }\end{array}$ \\
\hline 1 & 2,053 & 14,666 & 14,666 \\
\hline 2 & 1,640 & 11,711 & 26,378 \\
\hline 3 & 1,328 & 9,489 & 35,867 \\
\hline 4 & 1,248 & 8,912 & 44,778 \\
\hline 5 & 1,102 & 7,872 & 52,651 \\
\hline 6 & 1,049 & 7,495 & 60,146 \\
\hline 7 & 0,958 & 6,846 & 66,992 \\
\hline 8 & 0,936 & 6,686 & 73,678 \\
\hline 9 & 0,794 & 5,668 & 79,346 \\
\hline 10 & 0,706 & 5,044 & 84,390 \\
\hline 11 & 0,647 & 4,624 & 89,014 \\
\hline 12 & 0,623 & 4,451 & 93,464 \\
\hline 13 & 0,469 & 3,349 & 96,813 \\
\hline 14 & 0,446 & 3,187 & 100,000 \\
\hline
\end{tabular}

En la tabla 5 de componentes y variables, se destacan las variables que más influyen por componentes, como son: la cantidad de animales que se venden, las condiciones higiénicas para el sacrificio, aportes económicos por la venta, tipo de comprador y la rentabilidad, entre otras variables.

Otros aspectos que ilustran los bajos indicadores económicos de la producción caprino-ovino en el municipio de Riohacha, se reflejan en las figuras 2 y 3 .

Figura 2. Asistencia técnica que reciben o no los productores indígenas Wayuu

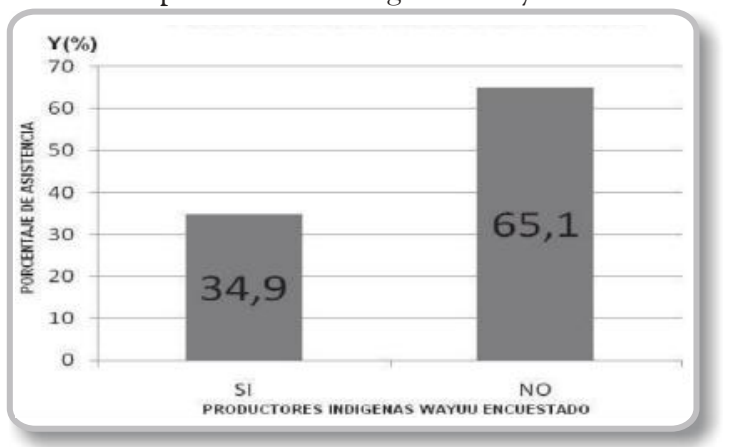


Análisis multivariado de indicadores socioeconómico-productivos del ganado caprino-ovino en el departamento de La Guajira, Colombia

Tabla 5. Matriz de componentes y variables.

\begin{tabular}{|l|c|c|c|c|c|c|c|}
\hline \multicolumn{1}{|c|}{ Variable } & \multicolumn{7}{|c|}{ Componente } \\
\cline { 2 - 8 } & 1 & 2 & 3 & 4 & 5 & 6 & 7 \\
\hline $\begin{array}{c}\text { Numero de hijos por } \\
\text { familias o castas. }\end{array}$ & $-0,383$ & 0,041 & 0,463 & 0,255 & 0,318 & 0,488 & $-0,019$ \\
\hline \multicolumn{1}{c}{ Familia o casta. } & $-0,179$ & $-0,274$ & $-0,356$ & $-0,323$ & 0,415 & $-0,039$ & $-0,381$ \\
\hline $\begin{array}{l}\text { Cantidad de animales } \\
\text { producidos para el } \\
\text { consumo humano. }\end{array}$ & $-0,320$ & $-0,428$ & 0,081 & $-0,112$ & 0,049 & $\mathbf{0 , 5 9 6}$ & 0,306 \\
\hline $\begin{array}{l}\text { Tipo de comprador de } \\
\text { animales producidos. }\end{array}$ & $-0,607$ & $\mathbf{- 0 , 6 0 4}$ & 0,092 & $\mathbf{0 , 4 7 6}$ & 0,235 & $-0,320$ & $-0,022$ \\
\hline $\begin{array}{c}\text { Preferencia por la venta } \\
\text { al consumidor. }\end{array}$ & 0,399 & $-0,325$ & $\mathbf{0 , 5 3 1}$ & 0,047 & $-0,026$ & $-0,070$ & $-0,232$ \\
\hline $\begin{array}{l}\text { Tipo de transporte para el } \\
\text { traslado de los animales }\end{array}$ & $-0,110$ & 0,509 & 0,041 & 0,454 & 0,224 & $-0,024$ & $-0,263$ \\
\hline $\begin{array}{c}\text { Cantidad de animales } \\
\text { que vende mensualmente. }\end{array}$ & $\mathbf{- 0 , 5 7 6}$ & 0,373 & $-0,124$ & 0,128 & 0,036 & $-0,269$ & 0,150 \\
\hline $\begin{array}{l}\text { Precio de venta de los } \\
\text { animales. }\end{array}$ & $-0,365$ & 0,266 & 0,305 & $-0,211$ & $\mathbf{0 , 5 5 0}$ & $-0,217$ & 0,044 \\
\hline $\begin{array}{l}\text { Tipos de indicadores qu e } \\
\text { determinan la compra } \\
\text { venta de los animales. }\end{array}$ & 0,458 & 0,499 & $-0,046$ & $-0,386$ & 0,147 & 0,208 & 0,447 \\
\hline $\begin{array}{l}\text { Tipos de indicadores que } \\
\text { se consideran al } \\
\text { inspeccionar } \\
\text { animales. }\end{array}$ & $-0,682$ & $-0,091$ & 0,473 & $-0,105$ & $-0,298$ & $-0,319$ & 0,218 \\
\hline $\begin{array}{l}\text { Asistencia técnica que } \\
\text { reciben. }\end{array}$ & 0,499 & $-0,156$ & $-0,374$ & 0,327 & 0,186 & 0,120 & $-0,130$ \\
\hline $\begin{array}{l}\text { Condiciones higiénicas } \\
\text { de los centros de } \\
\text { sacrificio de los } \\
\text { animales. }\end{array}$ & $\mathbf{0 , 5 0 5}$ & 0,391 & 0,259 & 0,342 & $-0,055$ & 0,174 & $-0,069$ \\
\hline $\begin{array}{c}\text { Rentabilidad de la } \\
\text { comercialización de los } \\
\text { animales. }\end{array}$ & 0,365 & $-0,003$ & $-0,221$ & 0,239 & 0,340 & $-0,104$ & $\mathbf{0 , 7 0 6}$ \\
\hline $\begin{array}{l}\text { Aporte económico por la } \\
\text { venta del ganado menor. }\end{array}$ & $\mathbf{0 , 5 3 0}$ & $-0,117$ & 0,315 & $-0,358$ & 0,391 & $-0,162$ & 0,044 \\
\hline
\end{tabular}

Figura 3. Ventas mensuales del ganado caprino - ovino.

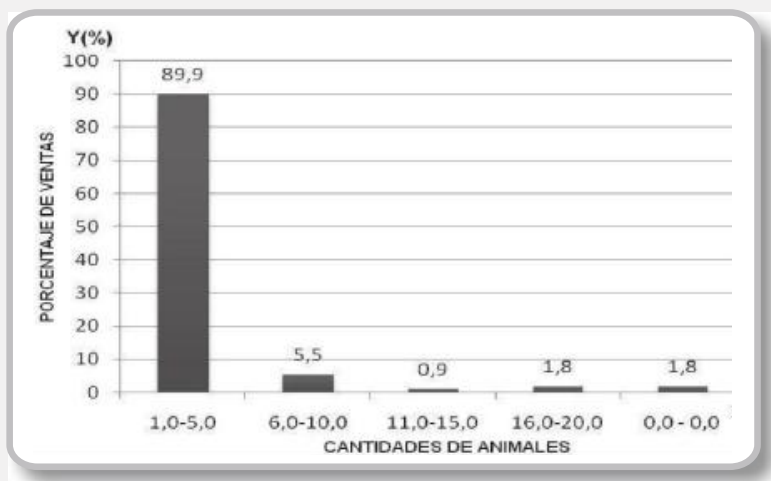

La tabla 6, presenta los fundamentales estadígrafos de posición y dispersión de las variables o indicadores productivos, excepto las variables: (6) épocas del año en que más se enferma el ganado menor y (7) tipos de enfermedades que afecta a los animales, que son categóricas nominales. Se destaca en estos resultados que es bajo, el estimado de animales con problemas genéticos y de lactancia, sin embargo la producción media de litros de leche por día animal es de 0,61 litros, produciendo el $25 \%$ de los animales hasta 0,25 litros/día/animal, el 50\% hasta 0,50 litros/día/ animal y el $75 \%$ hasta 1 litros/día/animal. Se aprecia que este indicador es extremadamente bajo, lo que está dado por las condiciones tan precarias en que se desarrolla esta producción. Se presenta muy elevada la variabilidad de los 
Tabla 6. Estadígrafo de posición y dispersión del número de las variables.

\begin{tabular}{|l|c|c|c|c|c|c|c|}
\hline & $\begin{array}{c}\text { Promedio } \\
\text { de vida } \\
\text { del } \\
\text { ganado. }\end{array}$ & $\begin{array}{c}\text { Promedio } \\
\text { de vida } \\
\text { para el } \\
\text { sacrificio }\end{array}$ & $\begin{array}{c}\mathrm{N}^{\mathbf{0}} \text { de } \\
\text { hebras con } \\
\text { problemas } \\
\text { genéticos. }\end{array}$ & $\begin{array}{c}\mathrm{N}^{\mathbf{0}} \text { de } \\
\text { caprinos } \\
\text { ovinos- } \\
\text { con } \\
\text { problemas } \\
\text { de } \\
\text { lactancia. }\end{array}$ & $\begin{array}{c}\text { Litros de } \\
\text { leche } \\
\text { diarios } \\
\text { obtenidos. }\end{array}$ & $\begin{array}{c}\mathrm{N}^{\text {o }} \text { promedio } \\
\text { de animales } \\
\text { muertos por } \\
\text { enfermedades }\end{array}$ & $\begin{array}{c}\mathrm{N}^{\mathbf{0}} \text { de } \\
\text { cabras en } \\
\text { ordeño }\end{array}$ \\
\hline $\begin{array}{l}\text { Media } \\
\text { aritmética }\end{array}$ & 5,41 & 10,90 & 2,61 & 3,11 & 0,61 & 8,47 & 7,45 \\
\hline $\begin{array}{l}\text { Mediana } \\
\text { (2do. } \\
\text { Cuartil) }\end{array}$ & 5,00 & 11,00 & 2,00 & 2,00 & 0,50 & 6,00 & 5,00 \\
\hline Moda & 3,00 & 12,00 & 0,00 & 0,00 & 1,00 & 5,00 & 0,00 \\
\hline Varianza & 8,35 & 10,82 & 11,60 & 14,81 & 0,17 & 43,24 & 121,95 \\
\hline $\begin{array}{l}\text { Desviación } \\
\text { típica }\end{array}$ & 2,89 & 3,29 & 3,41 & 3,85 & 0,41 & 6,58 & 11,04 \\
\hline Mínimo & 1,50 & 4,00 & 0,00 & 0,00 & 0,00 & 0,00 & 0,00 \\
\hline Máximo & 15,50 & 24,00 & 20,00 & 25,00 & 1,00 & 30.00 & 65,00 \\
\hline Rango & 14,00 & 20,00 & 20,00 & 25,00 & 1,00 & 30,00 & 65,00 \\
\hline $\begin{array}{l}\text { Primer } \\
\text { cuartil }\end{array}$ & 3,00 & 8,75 & 0,00 & 0,00 & 0,25 & 4,00 & 0,50 \\
\hline $\begin{array}{l}\text { Tercer } \\
\text { cuartil }\end{array}$ & 6,00 & 12,25 & 3,00 & 5,00 & 1,00 & 10,00 & 8,00 \\
\hline $\begin{array}{l}\text { Coeficiente } \\
\text { de } \\
\text { variación }\end{array}$ & $\mathbf{5 3 , 4 2 \%}$ & $\mathbf{3 0 , 1 9 \%}$ & $\mathbf{1 3 0 , 2 9 \%}$ & $\mathbf{1 2 3 , 6 2 \%}$ & $\mathbf{6 7 , 6 7 \%}$ & $\mathbf{7 7 , 6 7 \%}$ & $\mathbf{1 4 8 , 1 4 \%}$ \\
\hline
\end{tabular}

animales con problemas genéticos, lactancia y el número de cabras por ordeño, aunque en ninguno de los indicadores analizados la dispersión presenta bajos niveles, lo que resulta lógico por lo antes señalado.

La tabla 7 recoge los resultados del contraste de Bartlett, la cual indica que es muy improbable $(\mathrm{p}=0,001)$ que la matriz de correlación de las variables, sea una matriz identidad.

Tabla 7. Resultados del contraste de esfericidad de Bartlett.

\begin{tabular}{|c|c|c|}
\hline \multirow{2}{*}{$\begin{array}{c}\text { Prueba de esfericidad } \\
\text { de Bartlett }\end{array}$} & $\begin{array}{c}\text { Chi-cuadrado } \\
\text { aproximado }\end{array}$ & 68,675 \\
\cline { 2 - 3 } & Grados de libertad & 36 \\
\cline { 2 - 3 } & Significación. & 0,001 \\
\hline
\end{tabular}

En la tabla 8, de las 9 variables productivas se seleccionaron 4 componentes principales, que representan el 61,821\% de la variación total ya que tienen un auto valor superior a 1.0.

Tabla 8. Resultados de la aplicación del análisis de componentes principales.

\begin{tabular}{|c|c|c|c|}
\hline \multirow{2}{*}{ Componente } & \multicolumn{3}{|c|}{ Autovalores iniciales } \\
\hline & & & \\
& Total & \% de la varianza & \% acumulado \\
\hline 1 & 1,831 & 20,341 & 20,341 \\
\hline 2 & 1,331 & 14,790 & 35,131 \\
\hline 3 & 1,265 & 14,052 & 49,183 \\
\hline 4 & 1,137 & 12,638 & 61,821 \\
\hline 5 & 0,997 & 11,076 & 72,897 \\
\hline 6 & 0,789 & 8,771 & 81,668 \\
\hline 7 & 0,674 & 7,486 & 89,154 \\
\hline 8 & 0,539 & 5,987 & 95,141 \\
\hline 9 & 0,437 & 4,859 & 100,000 \\
\hline
\end{tabular}


Análisis multivariado de indicadores socioeconómico-productivos del ganado caprino-ovino en el departamento de La Guajira, Colombia

En la tabla 9, se aprecia que las variables que más inciden, son las de tipo genéticoproductivo y salud de los animales.

Tabla 9. Matriz de componentes y variables productivas.

\begin{tabular}{|c|c|c|c|c|c|}
\hline \multirow[b]{2}{*}{ Variable } & \multicolumn{5}{|c|}{ Componente } \\
\hline & 1 & 2 & 3 & 4 & 5 \\
\hline Promedio de vida del ganado & 0,250 & 0,586 & 0,206 & 0,414 & 0,484 \\
\hline $\begin{array}{l}\text { Promedio de vida para el } \\
\text { sacrificio }\end{array}$ & 0,178 & 0,138 & 0,454 & 0,616 & $-0,476$ \\
\hline $\begin{array}{c}\text { Número de hembras con } \\
\text { problemas genéticos (preñez) }\end{array}$ & 0,523 & $-0,523$ & 0,034 & 0,287 & $-0,002$ \\
\hline $\begin{array}{l}\text { Número de caprino-ovino con } \\
\text { problemas de lactancia }\end{array}$ & 0,683 & $-0,252$ & 0,368 & $-0,093$ & $-0,045$ \\
\hline Litros de leche diarios obtenidos & 0,484 & 0,364 & $-0,330$ & $-0,210$ & 0,053 \\
\hline $\begin{array}{l}\text { Épocas del año en que más se } \\
\text { enferma el ganado }\end{array}$ & $-0,146$ & 0,266 & 0,706 & $-0,295$ & 0,325 \\
\hline $\begin{array}{l}\text { Tipos de enfermedades que los } \\
\text { afectan }\end{array}$ & $-0,169$ & $-0,526$ & $-0,101$ & 0,375 & 0,635 \\
\hline $\begin{array}{l}\text { Número promedio de animales } \\
\text { muertos por enfermedades }\end{array}$ & 0,441 & 0,358 & $-0,503$ & 0,252 & 0,055 \\
\hline Número de cabras en ordeño & 0,721 & $-0,153$ & 0,092 & $-0,400$ & 0,139 \\
\hline
\end{tabular}

En la tabla 10, se muestra el análisis de la época del año en que más se enferma el ganado menor y los tipos de enfermedades que los afectan (8), también se observa que existe dependencia (con $\mathrm{p}=0,0020$ ), entre estas dos variables, y la mayor frecuencia de las enfermedades es en la época de invierno.

Tabla 10. Análisis de la relación entre el tipo de enfermedad y la época.

\begin{tabular}{|c|c|c|c|c|c|c|c|c|}
\hline & \multicolumn{7}{|c|}{ Tipo de enfermedad } \\
\hline Época & $\mathbf{1}$ & $\mathbf{2}$ & $\mathbf{3}$ & $\mathbf{4}$ & $\mathbf{5}$ & $\mathbf{6}$ & $\mathbf{7}$ & $\mathbf{8}$ \\
\hline Verano & 1 & 0 & 0 & 0 & 0 & 5 & 0 & 1 \\
\hline Invierno & 16 & 3 & 10 & 13 & 2 & 7 & 16 & 14 \\
\hline
\end{tabular}

Estadístico Chi-cuadrado $=22,56 \mathrm{p}=0,0020$

Enfermedades: 1.-Parasitosis, 2.-Mastitis, 3.-Enfermedades carenciales, 4.-Enfermedades bacterianas, 5.-Estomatitis vesicular, 6.Piodermitis, 7.-Enfermedades diarreicas agudas y 8.-Otras enfermedades.
La experiencia acumulada sobre el análisis estadístico en las investigaciones que usan encuestas, dado por Ojeda (2003) en trabajos realizados desde 1990, indican que los análisis de datos de encuestas van desde la obtención de estadísticos descriptivos como promedios, frecuencias, porcentajes, varianzas entre otros, hasta el uso de técnicas multivariadas, como análisis discriminante, análisis Factorial, etc, coincidiendo con lo señalado por Vara y Rubio (2005). Una de las formas de análisis ampliamente utilizadas para determinar si efectivamente una variable influye o no sobre otra en tablas de doble entrada, es la que se realiza sobre la base de la llamada regla de Zeizel (Ibarra, 2005).

Trabajos realizados en el campo agrario basados en la recogida de información por encuestas, lo han reportado Álvarez (1980) y Ramírez (2002), en el cultivo de los cítricos en Cuba y en fincas ganaderas en Colombia, respectivamente.

\section{Conclusiones}

Se puede señalar que resulta bajo, el estimado de animales con problemas genéticos, lactancia y salud animal, presentando una elevada variabilidad, además contrastando con producciones de leche en litros/día/ animal extremadamente bajas, lo que está dado por las condiciones tan precarias en que se desarrolla esta producción.

El Análisis de componentes principales, mostró que las variables que más inciden son fundamentalmente los indicadores de compraventa, las de tipo genético-productivo y de salud de los animales, existiendo dependencia entre la época del año y el tipo de enfermedad que padecen, predominando la época de invierno. Diciembre 2008 ISSN 0122-820X

(n)

Respuestas

Año 13

No. 2

(1) 
fin de visionar un fortalecimiento económico y social de la producción-comercialización del ganado caprino-ovino, buscando la mayor calidad alimentaría, con mayores estándares sanitarios que garanticen que no existe riesgo al consumidor, y que se traduzca en un creciente y más justo intercambio comercial de la región.

\section{Bibliografía}

1. Álvarez, M. M. 1980. Una encuesta global en la rama agropecuaria como instrumento para la investigación. Tesis para optar el grado de Doctor en Ciencias Agrícolas. ISCAH. La Habana, 126 p.

2. Berenson, M; Levine, D.M. 1996. Estadística para Administración Economía. Concepto y Aplicaciones. Mc Graw Hill. I.S.B.N. 968-422-713-2.

3. Bouza, C.N. y Sistachs, V. 2002. Estadística. Teoría básica y ejercicios. Editorial Félix Varela. La Habana. Cuba. 406p.

4. Departamento Administrativo Nacional de Estadística en Colombia. DANE. 2004.

5. Fernández, M.V y Ruiz, F.N. Muestreo estadístico en poblaciones finitas. 2006. Septem ediciones.

6. García, M. 2005. La Encuesta. Selección de lecturas de Metodología, Métodos y Técnicas de Investigación Social II. Editorial Félix Varela, La Habana, Cuba. 245p.

7. Hair, J. F; Anderson, R. E; Tatham, R. L; Black, W. C. 1999. Análisis Multivariante. Prentice Hall Iberia. Madrid. 799p.

8. Härdle, W. and Simar, L. 2003. Applied Multivariate Statistical Analysis. MD TECH, 486p.

9. Hernández-Sampieri, R.; Collados, C. y Baptista, P. 2003. Metodología de la Investigación. Mc Graw-Hill Interamericana Editores S.A. Tercera Edición. México. 684p.

10. Ibarra, F. 2005. El procesamiento y análisis de la información. Selección de lecturas de Metodología, Métodos y Técnicas de Investigación Social II. Editorial Félix Varela, La Habana, Cuba. 245p.

11. Lind, R.D; Mason, W.G; Marchal, L.M. 2001. Estadística para administración y economía. Ed. Irwin Mc Graw-Hill.

12. Márquez, J. 2003-2006. Protección a la Producción de Caprinos en La Guajira. Instituto Colombiano Agropecuario. ICA.

13. Martínez, B.C. Estadística y muestreo. 2003. ISBN 958-64-8283-9.

14. Ojeda, M. M. 2003. La Inferencia en el Muestreo de poblaciones finitas y el análisis de datos de encuestas. Revista Investigación Operacional.24 (2):143-152.

15. Ramírez, B. L. 2002. Diagnóstico ambiental y alternativas de desarrollo sostenible en fincas ganaderas establecidas en la Amazonia colombiana. Tesis en opción al Grado Científico de Doctor en Ciencias Veterinarias. UNAH, Cuba.

16. Statistical Graphics Corp. 2001. Statgraphics Plus 5.1 for Windows.

17. Tamayo, M. 2003. El Proceso de Investigación Científica. Limusa-Noriega Editores. México. 440p.

18. The Apache Software Foundation. 2004. SPSS 13.0 for Windows.

19. Unidad de asuntos indígenas de Riohacha, departamento de La Guajira. 2006.

20. Vara, M.J. ; Rubio, I. 2005. La Encuesta. Selección de lecturas de Metodología, Métodos y Técnicas de Investigación Social II. Editorial Félix Varela, La Habana, Cuba. $245 \mathrm{p}$. 